\title{
Clusterin: Its Relevance to Antitumour Drug Sensitivity
}

\section{Maximino Redondo*}

Department of Biochemistry, Hospital Costa del Sol, University of Málaga, CIBER ESP, Spain

Chemotherapy resistance is a major problem in disease management and the principal factor underlying most cancer deaths. Therefore, a primary goal in cancer research is to develop new ways of inhibiting cancer growth, in part by improving the effectiveness of existing cancer regimens. Resistance to cancer treatment is known to be mediated, at least in part, by the enhanced expression of cell survival proteins that facilitate tumour progression. In this respect, the clusterin protein (CLU) has drawn much attention because of its association with tumorigenesis and progression and its implication in two contrasting functions, survival and apoptosis, which are carry out by two different forms (secretory, s-CLU and nuclear, n-CLU, respectively). Most authors agree that tumour cell survival is related to the overexpression of sCLU and the loss of n-CLU [1]. It is noteworthy that only the cytoplasmic/secretory CLU form (sCLU) and not the nuclear form (nCLU) is expressed in aggressive late stage tumours, which is in line with its anti-apoptotic function. At present, there is no doubt as to the dual forms and functions of CLU [2].

Many reports have confirmed in cancer cell lines the chemosensitivity to cytotoxic treatment after anti-CLU therapy with small interfering RNA (siRNA) or antisense oligonucleotides. Thus, sCLU expression has been shown to lead to broad-based resistance to unrelated chemotherapeutic agents such as paclitaxel/docexatel, doxorubicin, cisplatin, etoposide and camphothecin. For this reason, much attention is being paid to sCLU in clinical trials [3,4]. However, when using siRNA, great care must be taken to reduce sCLU levels without affecting those of nCLU. siRNA knockdown sCLU levels enhance cytotoxic agent efficacy, whereas siRNA against nCLU confers cytoprotection against a variety of chemotherapeutics. Thus, the target sequence of the antisense oligonucleotides used in these trials (OGX11 ) is exon 2 of the CLU gene, which is common to all the CLU isoform transcripts that have been identified. Let us recall that in the most prevalent tumours, $\mathrm{n}$-CLU is rarely detected by immunohistochemistry $[5,6]$. In phase II trials, the combination of anti-CLU therapy (OGX11) and chemotherapeutic drugs is well tolerated and clinical activity has been observed, including pain palliation in prostate carcinomas [7]. It is too soon to predict the efficacy of anti-CLU treatment in cancer patients, but the promise of disrupting s-CLU to achieve better chemosensitivity to established drugs remains an attractive goal.

Concerning other treatment modalities such as antihormone therapy, several reports have associated CLU overexpression with resistance to antihormone therapy in breast and prostate carcinomas. The observation that by down-regulating CLU in oestrogen-receptor positive cell lines made sensitive these cells to antioestrogen treatment $[8,9]$ represents an interesting contribution as CLU expression may be determined together with oestrogen receptors before starting treatment with antioestrogens. Such results, if confirmed in clinical trials, could provide a rationale for modulating sensitivity to antioestrogens by combining traditional therapy with strategies targeting CLU in vivo.

While glucocorticoids are routinely used in the clinical application of chemotherapy, in glandular cells they protect against the apoptotic signals evoked by cytokines, cAMP, tumour suppressors and death genes. It is well known that the anti-apoptotic effect of glucocorticoids is exerted by the modulation of survival genes such as $\mathrm{Bcl}-2, \mathrm{Bcl}-\mathrm{x}(\mathrm{L})$ and NFkappaB, in a cell type-specific manner [10]. In breast cancer, dexamethasone induces the expression of CLU and, importantly, inhibits chemotherapy-induced cytotoxicity despite the previous administration of dexamethasone [9].

Overall, it is now evident that s-CLU is a powerful mediator of cell survival that can block the effects of almost all known therapeutic agents. However, as CLU may have different patterns of expression in different tissues, an analysis of CLU expression may help to better discriminate patients who could benefit from anticlusterin treatment. Future research should focus on identifying CLU form specific epitopes and on the production of CLU form specific antibodies. Understanding the molecular regulation and structure of CLU isoforms is essential, especially as the s-CLU: n-CLU ratio seems to be critical to cancer survival and progression.

\section{References}

1. Pucci S, Bonanno E, Pichiorri F, Angeloni C, Spagnoli LG (2004) Modulation of different clusterin isoforms in human colon tumorigenesis. Oncogene 23: 22982304.

2. Wei L, Xue T, Wang J, Chen B, Lei Y, et al. (2009) Roles of clusterin in progression, chemoresistance and metastasis of human ovarian cancer. Int $J$ Cancer 125: 791-806.

3. Chi KN, Zoubeidi A, Gleave ME (2008) Custirsen (OGX-011): a secondgeneration antisense inhibitor of clusterin for the treatment of cancer. Expert Opin Investig Drugs 17: 1955-1962.

4. Chi KN, Hotte SJ, Yu EY, Tu D, Eigl BJ, et al. (2010) Randomized phase II study of docetaxel and prednisone with or without OGX-011 in patients with metastatic castration-resistant prostate cancer. J Clin Oncol. 28: 4247-4254.

5. Redondo M, Tellez T, Roldan MJ (2009) The role of clusterin (CLU) in malignant transformation and drug resistance in breast carcinomas Adv Cancer Res. 105: $21-43$.

6. Redondo M, Rodrigo I, Alcaide J, Tellez T, Roldan MJ, et al. (2010) Clusterin expression is associated with decreased disease-free survival of patients with colorectal carcinomas. Histopathology 56: 932-936.

7. Saad F, Hotte S, North S, Eigl B, Chi KN, et al. Randomized Phase 2 Trial of Custirsen (OGX-011) with Docetaxel or Mitoxantrone in Patients with Metastatic Castrate-Resistant Prostate Cancer: CUOG Trial P06c. Clin Cancer Res. 17 5765-5773.

8. Toffanin S, Daidone MG, Miodini P, De Cecco L, Gandellini P, et al. (2008) Clusterin: a potential target for improving response to antiestrogens. Int $J$ Oncol. 33: 791-798.

9. Redondo M, Téllez T, Roldan MJ, Serrano A, García-Aranda M, et al. (2007) Anticlusterin treatment of breast cancer cells increases the sensitivities of chemotherapy and tamoxifen and counteracts the inhibitory action of dexamethasone on chemotherapy-induced cytotoxicity. Breast Cancer Res. 9 : R86.

10. Amsterdam A, Sasson R (2002) The anti-inflammatory action of glucocorticoids is mediated by cell type specific regulation of apoptosis. Mol Cell Endocrinol. 189: $1-9$.

*Corresponding author: Maximino Redondo, Department of Biochemistry, Hospital Costa del Sol, University of Málaga, CIBER ESP, Carretera de Cádiz km 187, 29600, Marbella, Málaga, Spain, E-mail: mredondo@hcs.es

Received September 08, 2011; Accepted Sepetmber 10, 2011; Published November 12, 2011

Citation: Redondo M (2011) Clusterin: Its Relevance to Antitumour Drug Sensitivity. Clin Exp Pharmacol 1:e101. doi:10.4172/2161-1459.1000e101

Copyright: (c) 2011 Redondo M. This is an open-access article distributed under the terms of the Creative Commons Attribution License, which permits unrestricted use, distribution, and reproduction in any medium, provided the original author and source are credited. 